\title{
Determinación de la presencia de fatiga mecánica en materiales cerámicos
}

\author{
D. CASELLAS, M.M. NAGL, M. VÉLEZ*, L. LLANES Y M. ANGLADA \\ Departamento de Ciencia de los Materiales e Ingeniería Metalúrgica. E.T.S.I.I.B. (U.P.C.). Avda. Diagonal, 647, 08028 Barcelona \\ *Dept. de Ciencia de Materiales, Universidad Simón Bolívar.Caracas 1080A, Venezuela
}

\begin{abstract}
Los materiales cerámicos son susceptibles al crecimiento de fisuras por la acción combinada del medio ambiente y la aplicación de carga (fatiga estática). Por otra parte, el crecimiento subcrítico de fisuras en cerámicas también puede ser producido por la aplicación de cargas fluctuantes (fatiga mecánica). Este fenómeno no se observa en vidrios ni en cerámicas monocristalinas, pero si en muchos de los llamados materiales cerámicos avanzados. En este trabajo se describe una metodología utilizada para detectar la presencia de fatiga mecánica en materiales cerámicos, basada en la obtención de estimaciones de la vida a fatiga del material bajo cargas fluctuantes y su comparación con los resultados experimentales bajo carga constante. Este método se ha aplicado a fisuras naturales y de indentación en algunos materiales cerámicos (mullita, alúmina y ZTA).
\end{abstract}

Palabras clave: Fatiga cíclica, fatiga estática, fisuras naturales, fisuras de indentación.

\section{Mechanical fatigue detection on ceramic materials}

It is well known that ceramic materials are highly susceptible to environmental assisted subcritical crack growth. In addition, this subcritical crack growth could be also related to cyclic loading (mechanical fatigue). This type of behaviour is not observed either in glasses nor in traditional ceramics. However this phenomena is clearly active in many advanced ceramics. The aim of this work is to obtain a procedure for detecting the presence of cyclic fatigue in ceramic materials. This procedure attempts to obtain cyclic fatigue life estimations and to compare it with the static loading experimental results. Through this comparisons it is possible to asses whether the cyclic fatigue is acting or not. Finally some calculations are conducted in ceramic materials (alumina, mullite and ZTA) with natural and indentation flaws.

Key words: Cyclic fatigue, static fatigue, natural flaws, indentation flaws.

\section{INTRODUCCIÓN}

\subsection{Materiales cerámicos avanzados}

Los materiales cerámicos son candidatos para varias aplicaciones estructurales, puesto que presentan buena resistencia frente a la corrosión en medios agresivos, mantienen sus propiedades mecánicas a temperaturas elevadas (superiores a los $1000{ }^{\circ} \mathrm{C}$ ) y ofrecen una buena relación resistencia/peso. En cambio, el principal problema es su fragilidad intrínseca y la dificultad en controlar y detectar los defectos producidos durante su procesamiento. Con el fin de aumentar la tenacidad en estos materiales se han desarrollado los denominados materiales cerámicos avanzados o de ingeniería. Éstos pueden tener valores de tenacidad de fractura $\left(\mathrm{K}_{\mathrm{IC}}\right)$ superiores a $10 \mathrm{MPa}$ $\mathrm{m}^{1 / 2}(1-4)$, y de esta manera aumentar sus posibilidades como materiales estructurales. Esta nueva aplicación obliga a estudiar y caracterizar su comportamiento bajo los principales tipos de solicitación: aplicación de una carga constante o aplicación de cargas fluctuantes. El primero de ellos es un fenómeno bastante bien conocido y reportado. En cambio, la aplicación de cargas cíclicas abre un nuevo campo de estudio, puesto que los materiales cerámicos avanzados, a diferencia de los vidrios y cerámicas monolíticas, presentan degradación debido a las cargas fluctuantes aplicadas. El efecto de cada uno de estos casos se estudia en detalle en los siguientes apartados.

\subsection{Comportamiento bajo cargas constantes}

En materiales cerámicos y en vidrios se observa que la aplicación de una carga constante produce una disminución de la resistencia a fractura $\left(\sigma_{\mathrm{F}}\right)$ en función del tiempo. Este fenómeno es conocido desde hace años (5-8) y en la literatura cerámica recibe el nombre de fatiga estática. Su causa es la degradación que ejerce sobre el material el agua ambiental (5-15), facilitando la propagación de fisuras preexistentes. Esto se pone de manifiesto viendo la influencia que tiene el grado de humedad en la disminución de $\sigma_{\mathrm{F}}$. Así, en agua el efecto de fatiga estática es mucho más acusado que en aire y más que en nitrógeno seco o en vacío (8-14). Dado que muchos materiales cerámicos presentan una fase vítrea intergranular procedente de los procesos de sinterización, algunos autores proponen mecanismos basados en la interacción de esta fase vítrea con el agua 
ambiental (16) para explicar la degradación observada. Según este modelo, el par de electrones deslocalizados del agua ataca los enlaces Si-O, proceso facilitado por la deformación de éstos (alrededor del 20\%) debido a la carga aplicada. Este modelo también explica que en medios como el amoníaco o la hidracina se produzca fatiga estática mientras que no se observa para medios de nitrógeno, $\mathrm{CO}$ o acetonitrilo. Dado que en materiales cerámicos las fisuras son afiladas el acceso del agua a la punta de la fisura es difícil. Por eso se plantea un modelo de "cuña químico" (17) basado en la acción capilar sobre las moléculas de agua quimisorbidas que abren las caras de la fisura y permiten al agua ambiental degradar más fácilmente el material.

La detección experimental de este fenómeno se realiza a través de la curvas de $\sigma_{\mathrm{F}}$ en función del tiempo, conocidas como curvas S-t. En estas representaciones se observa una disminución de $\sigma_{\mathrm{F}}$ en función del tiempo, y se define un valor de tensión que no produce fractura para un tiempo dado, al cual se le denomina límite de fatiga estática $\left(\sigma_{\mathrm{th}, \mathrm{e}}\right)$.

El fenómeno de fatiga estática también se manifiesta en los denominados materiales cerámicos avanzados (5-15). En los materiales de base circona la acción del medio se centra en la degradación de la fase circona tetragonal, que es la responsable del aumento de tenacidad observado en estos materiales. Esta degradación se explica en términos de accesibilidad e interconexión de la fase circona tetragonal (9-15). Respecto a alúmina y materiales de base alúmina, como ZTA, el ataque del agua ambiental se centra en la fase vítrea intergranular que presentan $(9,15)$. En mullita la degradación por efecto del medio no viene influenciada por la fase amorfa intergranular, sino que es una característica intrínseca del material (43).

\subsection{Comportamiento bajo cargas cíclicas}

En materiales metálicos el comportamiento bajo este tipo de solicitaciones ha sido ampliamente estudiado desde finales del siglo pasado a raíz de roturas aparecidas en componentes de ferrocarril, donde la carga aplicada era inferior al límite elástico del material. Este fenómeno se denominó fatiga. En metales la presencia de fatiga se asocia al movimiento de dislocaciones y a la consiguiente deformación plástica que aparece en la punta de la fisura. Dado que en los materiales cerámicos tipo alúmina, mullita, etc., así como en vidrios, no existe ningún tipo de movimiento de dislocaciones a bajas temperaturas, durante mucho tiempo se creyó que el fenómeno de fatiga no existía en ellos (18). Sin embargo en la década de los 70 varios trabajos ya mencionan la presencia de fatiga en cerámicas (19, 20), que se denominó fatiga cíclica o mecánica para diferenciarla del crecimiento subcrítico por efecto del medio y una carga aplicada.

En materiales cerámicos, a diferencia de los metales, la microestructura del material no ofrece una resistencia intrínseca a la propagación de fisuras (21). Los mecanismos que producen aumento de tenacidad en cerámicas son extrínsecos y se basan en el concepto de apantallamiento de la punta de la fisura, desarrollando su acción detrás de ella, en su estela. Estos mecanismos reducen la fuerza motriz del crecimiento de fisura mediante la aplicación de esfuerzos de cierre de las caras de la fisura. Sin embargo, el aumento de tenacidad introducido en las cerámicas avanzadas lleva asociado una nueva situación, la susceptibilidad de estos materiales a sufrir fatiga cíclica (2226). La explicación se basa en que las cargas fluctuantes pueden degradar los mecanismos que actúan en la estela de la fisura y que son responsables del apantallamiento en su punta.
Los mecanismos extrínsecos de aumento de tenacidad se introducen a través de variaciones microestructurales. Los más comunes son (1-3):

- Transformación de fase de circona tetragonal: la circona $\left(\mathrm{ZrO}_{2}\right)$ presenta una transformación de fase tetragonal a monoclínica de tipo martensítico y que lleva asociado un aumento de volumen. Esta fase tetragonal se puede metaestabilizar a temperatura ambiente mediante óxidos metálicos $(\mathrm{MgO}, \mathrm{CaO}$, $\mathrm{CeO}_{2}$ ), de tal manera que la transformación de fase a temperatura ambiente tenga lugar sólo en presencia de un campo de tensiones aplicado. Así, el aumento de volumen se produce en la punta de una fisura y desarrolla esfuerzos compresivos que inducen su cierre.

- Unión de las caras de la fisura: en su propagación, la fisura puede dejar atrás obstáculos no fracturados (granos, precipitados fibras, whiskers, etc) que actúan como ligamentos que se oponen a la abertura de la fisura. El mecanismo adquiere más importancia a medida que aumenta el tamaño de estos ligamentos.

- Desvío en la trayectoria de la fisura: la introducción de un obstáculo que obligue a la fisura a desviar su trayectoria provoca que la tensión necesaria para seguir propagándose aumente. Estos obstáculos pueden ser partículas de segundas fases o granos cuando la propagación es intergranular.

- Microfisuración: la aparición de microfisuras en los bordes de grano es un fenómeno común debido a las tensiones residuales de origen térmico introducidas en la etapa de enfriamiento posterior a la sinterización. Estas microfisuras disipan energía cuando se forman y eso reduce la fuerza motriz de propagación de la fisura, además ocasionan un aumento de volumen que se traduce en un cierre de fisura.

De los mecanismos expuestos anteriormente los que producen mayores aumentos de $\mathrm{K}_{\mathrm{IC}}$ son los dos primeros (1). Estos mecanismos se ven afectados en mayor o menor grado por las cargas aplicadas. Así, el que más se degrada parece ser el de unión de las caras de la fisura (22-23), mientras que estudios recientes muestran que el mecanismo de transfor-mación de fase apenas sufre degradación (26).

\subsection{Detección del fenómeno de fatiga cíclica}

Para detectar la presencia del fenómeno de fatiga cíclica existen dos posibilidades. Una consiste en observar la propagación de una fisura grande bajo una carga constante y posteriormente bajo cargas cíclicas. A partir de estas observaciones se intenta establecer si hay diferencias significativas en la velocidad de propagación de fisura. En caso afirmativo se concluye que el material presenta fatiga cíclica (27). Una de las ventajas de este método es que se requiere pocas probetas, sin embargo solamente se puede aplicar a la propagación de fisuras grandes. Éstas se obtienen por procesos de prefisuración en probetas entalladas, y tienen dimensiones físicamente grandes (del orden de milímetros) (29). El fallo de componentes cerámicos procede de la propagación catastrófica de fisuras naturales, cuyas dimensiones son muy inferiores a las de las fisuras grandes comentadas anteriormente. El comportamiento de fisuras pequeñas y grandes en materiales cerámicos no es el mismo, sobretodo si existe un efecto de curva-R (3). Este efecto consiste en que la resistencia a la propagación de fisuras es función de su longitud, aumentando a medida que la fisura se hace más grande, hasta alcanzar el valor de $\mathrm{K}_{\mathrm{IC}}$. Este comportamiento de curva-R se da en los materiales cerámicos avanzados, en los cuales se han introducido mecanismos de aumento 
de tenacidad extrínsecos que actúan en la estela de la fisura, y por tanto adquieren mayor relevancia a medida que aumenta su longitud. Así, cuando las fisuras son pequeñas aún no han desarrollado su curva- $R$, y el comportamiento será muy diferente al de fisuras grandes, donde la resistencia al crecimiento de fisuras está más desarrollado. Por tanto, para extrapolar resultados obtenidos en propagación de fisuras grandes hay que ser cauteloso. El método propuesto en este trabajo para evaluar la presencia de fatiga cíclica se basa en estudiar la vida a fatiga de fisuras pequeñas, con tensiones residuales, pero sin curva-R.

\section{PROCEDIMIENTO EXPERIMENTAL}

\subsection{Materiales estudiados}

Los materiales estudiados en este trabajo son:

- Alúmina $\left(\mathrm{Al}_{2} \mathrm{O}_{3}\right)$ : según el tamaño de grano los valores de $\mathrm{K}_{\mathrm{IC}}$ y $\sigma_{\mathrm{F}}$ varían entre 2 y $6 \mathrm{MPa} \mathrm{m}^{1 / 2}$ y 200 y $800 \mathrm{MPa}$ respectivamente (4). El material estudiado en este trabajo es del $99.7 \%$ de pureza con una distribución bimodal de tamaños de grano $(1.6$ y $4.0 \mu \mathrm{m})$. Los valores de $\mathrm{K}_{\mathrm{IC}}$ y $\sigma_{\mathrm{F}}$ son $4.0 \mathrm{MPa} \mathrm{m}^{1 / 2}$ y 300 $\mathrm{MPa}$ respectivamente.

ZTA (Zirconia-Toughened-Alumina): es un material bifásico que consiste en partículas o agregados de circona $\left(\mathrm{ZrO}_{2}\right)$ tetragonal en una matriz de alúmina. Las propiedades mecánicas dependen de la fracción volumétrica de circona, obteniéndose valores de $\mathrm{K}_{\mathrm{IC}}$ comprendidos entre 4.0 y $6.0 \mathrm{MPa} \mathrm{m}^{1 / 2}$, y $\sigma_{\mathrm{F}}$ entre 300 y $600 \mathrm{MPa}$. El material estudiado presenta una fracción volumétrica de circona del $30 \%$, con unos tamaños de grano de $0.6 \mu \mathrm{m}$ para la matriz y $0.3 \mu \mathrm{m}$ para las partículas de circona. Los valores de $\mathrm{K}_{\mathrm{IC}}$ y $\sigma_{\mathrm{F}}$ son $5.0 \mathrm{MPa} \mathrm{m}^{1 / 2}$ y $500 \mathrm{MPa}$ respectivamente.

- Mullita $\left(3 \mathrm{Al}_{2} \mathrm{O}_{3} \cdot 2 \mathrm{SiO}_{2}\right)$ : e es una de las fases más comunes en materiales cerámicos tradicionales, tales como refractarios y porcelanas. Desde el punto de vista mecánico, la principal característica de los materiales de mullita es la conservación de su resistencia mecánica hasta temperaturas del orden de los $1200{ }^{\circ} \mathrm{C}$ y fundamentalmente su resistencia a la fluencia. La mullita estudiada presenta impurezas procedentes de los procesos de obtención. Éstas son mayoritariamente $\mathrm{ZrO}_{2}(>0.3 \%)$ $\mathrm{y}$, en menor grado, de tipo alcalino $(\sim 0.1 \%)$. El tamaño de grano medio es de $4.0 \mu \mathrm{m}$. El material presenta valores bajos de $\mathrm{K}_{\mathrm{IC}}$ y $\sigma_{\mathrm{F}}: \sim 2.0 \mathrm{MPa} \mathrm{m}^{1 / 2}$ y $\sim 250 \mathrm{MPa}$, respectivamente.

Más detalles sobre sus propiedades mecánicas, así como los procesos de elaboración se encuentran en trabajos previos (29, $39,43,45)$.

\subsection{Tipos de fisuras}

\subsubsection{FISURAS NATRUALES}

Cuando se intenta obtener $\sigma_{\mathrm{F}}$ de un material frágil, aparece una amplia dispersión en los resultados obtenidos. Si se dispone de suficientes muestras se puede encontrar un valor promedio de $\sigma_{\mathrm{F}}$ a partir de una curva de distribución de resultados, la cual se puede describir como una función tipo Weibull (30). Cuanto más ancha sea dicha curva de distribución más cuidadoso hay que ser a la hora de usar esos resultados. Varios factores afectan al ancho de esta distribución, tales como el tipo de procesamiento del material y el acabado superficial. Así, el estudio mecánico del comportamiento de grietas natu- rales, requiere del uso de parámetros estadísticos para obtener resultados fiables. Esto plantea un importante problema económico y de disponibilidad de material.

Basándose en lo expuesto anteriormente, se ve la necesidad de conocer y controlar el tamaño del defecto más grande, que será el que regirá el comportamiento a fractura del componente. Sin embargo, es difícil asegurar que todas las piezas procedentes de un mismo proceso de fabricación presenten la misma distribución de defectos y el mismo tamaño de defecto crítico. Para solucionar este inconveniente y poder estudiar las propiedades mecánicas, se suelen introducir defectos superficiales de dimensiones conocidas aplicando cargas con penetradores Vickers o Knoop (31-36).

\subsubsection{DEFECTOS SUPERFICIALES}

A diferencia de los metales, la aplicación de penetradores Vickers o Knoop en materiales frágiles induce la formación de fisuras superficiales. Los penetradores Knoop forman una sola fisura mientras que los Vickers dan un sistema con dos fisuras, en cada diagonal de la indentación. Con penetradores Vickers las fisuras superficiales quedan mejor definidas, aunque el campo de tensiones residuales que lleva asociado es más complicado que con penetradores Knoop.

Según la carga aplicada se formará un tipo u otro de fisura. Así, a partir de una carga de indentación crítica la fisura se inicia por debajo del punto de contacto debido a la generación de esfuerzos de tracción. Esta fisura subsuperficial se denomina fisura 'median' (figura 1a). Cuando se retira la carga la fisura media es inestable y crece hasta la huella de la superficie, dando lugar a una fisura radial, (figura 1b) (36). Éstas tienen morfología semielíptica y el factor de intensidad de tensiones que las describe está bien definido (37). En algunos materiales frágiles con superficies pulidas vastamente y tenacidad relativamente alta, la aplicación de cargas bajas produce fisuras radiales poco profundas y sin desarrollar completamente, conocidas como fisuras Palmqvist (figura 1c) (36). La generación de fisuras Palmqvist no es deseable para caracterizar el comportamiento mecánico porque el factor de intensidad de tensiones asociado es más complicado.

En los materiales estudiados con tenacidades no muy altas (alúmina, ZTA y mullita) las fisuras introducidas son de tipo radial, con morfología semielíptica (figura 1b) $(15,39)$. Las cargas aplicadas son relativamente altas para alúmina y ZTA, entre 198 y 394 N. En cambio, en mullita se han aplicado cargas más bajas, de $49 \mathrm{~N}$, debido a que su fragilidad permite obtener fisuras radiales con estas cargas. 


\section{DETERMINACIÓN DE LA PRESENCIA DE FATIGA CÍCLICA}

\subsection{Consideraciones generales}

En la aplicación de cargas cíclicas el material está expuesto a dos tipos de degradación. El primero por la acción combinada del medio y de la aplicación de una carga, es decir la fatiga estática asociada a la carga aplicada. El segundo tipo de degradación es debido a las cargas fluctuantes que, como se ha comentado anteriormente, pueden introducir mecanismos de degradación que produzcan la aparición de fatiga cíclica en el material.

Bajo solicitaciones estáticas el material experimenta crecimiento subcrítico de fisuras cuando el factor de intensidad de tensiones en la punta de la fisura es superior al valor umbral $\left(\mathrm{K}_{\text {the }}\right)$; y en correspondencia con ello, cuando la tensión aplicada es superior a $\sigma_{\text {th,e }}$. En la figura 2 se representa esta situación para los dos tipos de aplicación de carga: estática y cíclica, siendo la zona sombreada la que cumple la condición de crecimiento subcrítico $\left(K>K_{\text {th,e }}, \sigma>\sigma_{\text {th,e }}\right)$. Para comparar las dos aplicaciones de carga se ha igualado la tensión cíclica máxima $\left(\sigma_{\max }\right)$ a la aplicada para el caso estático $\left(\sigma_{\mathrm{e}}\right)$. De ahí, se puede observar que para solicitaciones cíclicas el material permanece menos tiempo en condiciones de crecimiento de fisura y el efecto del medio será menos importante en este caso. Por tanto, si un material no presenta fatiga cíclica su vida bajo cargas fluctuantes será mayor que para el caso estático. Así, para determinar si el comportamiento observado bajo cargas cíclicas responde a la presencia de fatiga cíclica, o simplemente es una manifestación del fenómeno de fatiga estática, basta con estimar la vida bajo cargas cíclicas suponiendo que sólo existe crecimiento subcrítico de fisuras asistido por el medio $\left(t_{c}\right)$ y compararla con el tiempo a fractura para el caso estático $\left(t_{\mathrm{e}}\right)$. Estos valores de tiempo se consiguen integrando la ecuación de velocidad de propagación de fisuras bajo carga constante, la cual se expresa comúnmente como:

$$
\frac{\mathrm{da}}{\mathrm{dt}}=\mathrm{AK}^{\mathrm{n}}
$$

donde a es la longitud de la fisura (semieje menor de la fisura semielíptica), t el tiempo, A y n constantes dependientes del material y K el factor de intensidad de tensiones que depende del tipo de fisura.

Los primeros estudios en que se realizó una estimación matemática de la vida a fatiga bajo cargas cíclicas se deben a Evans y Fuller (40). En dicho trabajo se dan los cocientes de tiempo de fractura $\left(\mathrm{t}_{\mathrm{c}} / \mathrm{t}_{\mathrm{e}}\right)$ para fisuras naturales a partir de ciertas aproximaciones matemáticas. En la actualidad, los avances en el campo del cálculo numérico por ordenador permiten integrar la ecuación (1) de manera relativamente sencilla. En los últimos años se han publicado algunos trabajos que abordan este tema para distintas solicitaciones y tipos de fisura (30, 41-42), aunque en ellos no se muestra la relación $t_{c} / t_{e}$ de manera directa para fisuras naturales y de indentación. Por eso, el objetivo del presente trabajo es calcular estos cocientes de tiempo para estos dos tipos de fisuras y poder evaluar a partir de ellos si existe fatiga cíclica en el material.

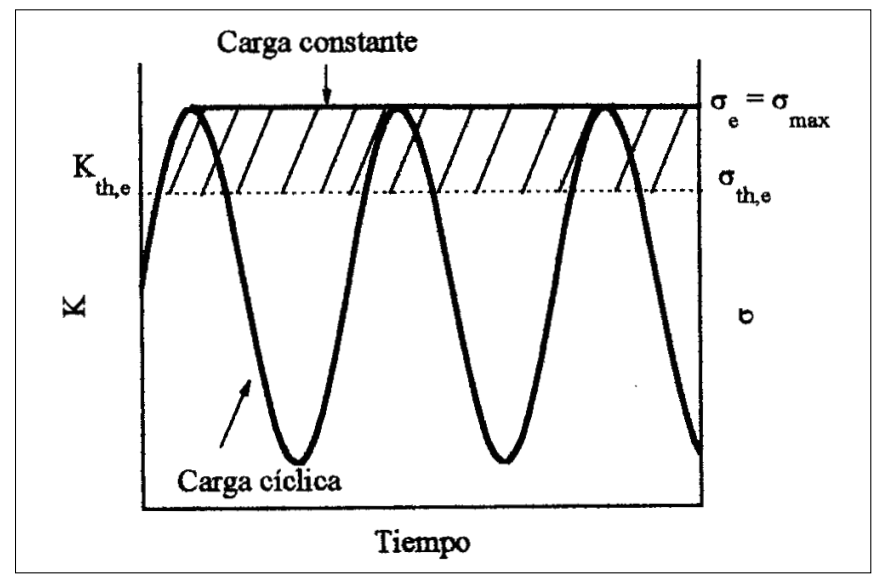

Figura 2: Esquema de los distintos tipos de solicitación y su influencia en el crecimiento subcrítico de fisuras.

\subsection{Fisuras naturales}

Para este caso, suponiendo que no existen tensiones residuales, el factor de intensidad de tensiones se puede expresar como:

$$
K=Y \sigma \sqrt{a}
$$

siendo $\mathrm{Y}$ un factor adimensional que depende de la geometría de la fisura y $\sigma$ la tensión aplicada.

\subsubsection{APLICACIÓN DE CARGA CONSTANTE}

En este caso el valor de $\sigma$ no varia con el tiempo, así la integración de la ecuación (1), usando la ecuación (2), permite obtener el tiempo de fractura bajo carga constante, $\mathrm{t}_{\mathrm{e}}$ :

$$
t_{\mathrm{e}}=\frac{\mathbf{f}}{\sigma_{\mathrm{e}}^{\mathrm{n}}}
$$

Siendo $\mathrm{f}$ una función que depende de A, Y, n y de la longitud de fisura crítica $\left(\mathrm{a}_{\mathrm{c}}\right)$ e inicial $\left(\mathrm{a}_{0}\right)$ :

$$
\mathrm{f}=\frac{1}{\mathrm{~A} \mathrm{Y}^{\mathrm{n}}} \frac{2-\mathrm{n}}{2}\left[\mathrm{a}_{\mathrm{c}}^{(2-\mathrm{n}) / 2}-\mathrm{a}_{0}^{(2-\mathrm{n}) / 2}\right]
$$

\subsubsection{APLICACIÓN DE CARGAS CÍCLICAS}

En este tipo de solicitación la carga aplicada varía sinusoidalmente con el tiempo (figura 3), y la relación de $\sigma$ con el tiempo adquiere la forma:

$$
\sigma(t)=\sigma_{m}+\sigma_{a} \sin (\omega t)
$$

Siendo $\sigma_{\mathrm{m}}$ la tensión media, $\sigma_{\mathrm{a}}$ la amplitud de tensiones y $\omega$ la frecuencia angular, relacionada con la frecuencia de los ensayos $(v)$ por $\omega=v 2 \pi$. 


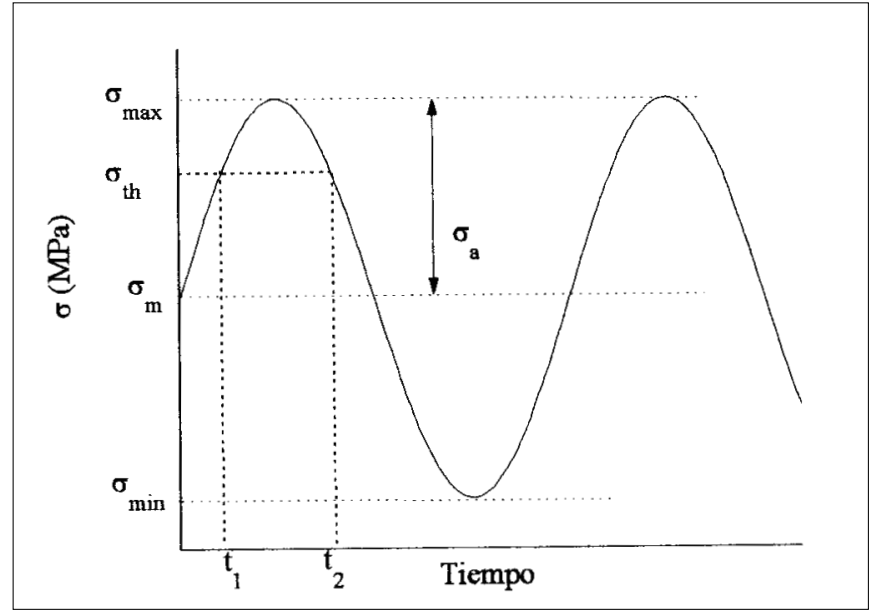

Figura 3: Esquema del tipo de aplicación de carga cíclica utilizado.

Un parámetro importante en este tipo de solicitación es el cociente entre las tensiones mínima y máxima $\left(\sigma_{\min } \mathrm{y} \sigma_{\max }\right.$ respectivamente) denominado razón de carga (R):

$$
\mathrm{R}=\frac{\sigma_{\min }}{\sigma_{\max }}
$$

Si el material sólo presenta fatiga cíclica la degradación depende de la magnitud y amplitud $\left(\sigma_{\mathrm{a}}\right)$ de las cargas aplicadas. Así, cuanto más separados estén los valores de $\sigma_{\max }$ y $\sigma_{\min }$ mayor será el daño ocasionado en el material. Esto significa que al disminuir $\mathrm{R}$ aumentará la degradación ejercida sobre el material. En cambio, si el material sólo presenta fatiga estática, el efecto es el contrario, puesto que el material se degrada cuando se cumplen las condiciones de crecimiento subcrítico de fisura $\left(\mathrm{K}>\mathrm{K}_{\text {the, }}, \sigma>\sigma_{\text {the }}\right)$. Por tanto, el material permanece más tiempo en condiciones de crecimiento de fisura a medida que aumenta el valor de $\mathrm{R}$ (figura 4).

La integración de la ecuación (1) se lleva a cabo en las condiciones de crecimiento de fisura, es decir, para valores de $\sigma$ superiores a $\sigma_{\text {the, }}$ (figura 3). A partir de estas condiciones y de la ecuación (5) se pueden obtener los tiempos de permanencia

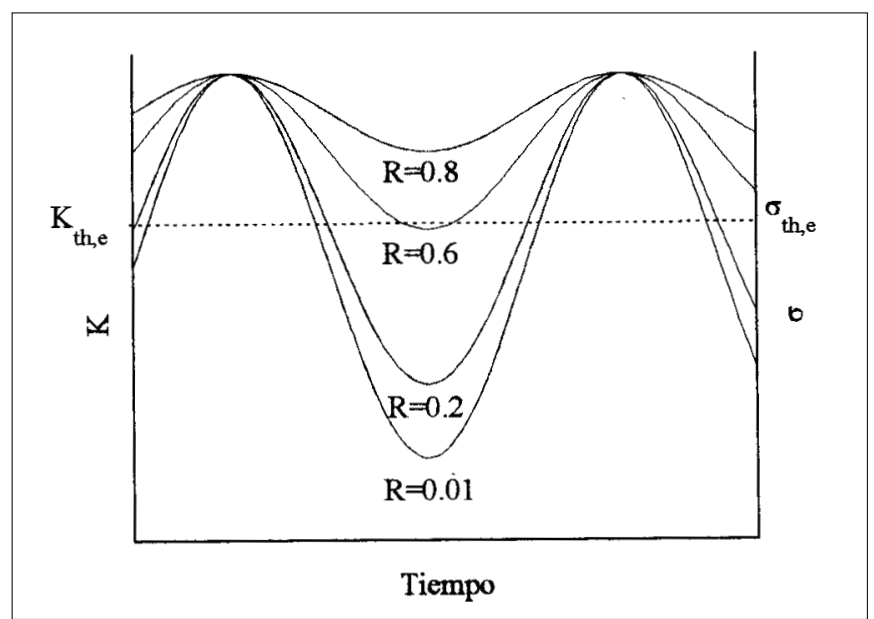

Figura 4: Influencia de la razón de carga (R) en el tiempo de permanencia bajo condiciones de crecimiento subcrítico de fisuras $\left(\mathrm{K}_{\text {th, }} \mathrm{f}^{\prime}\right.$ $\left.\sigma_{\text {th,e }}\right)$ por acción del medio. en condiciones de crecimiento de fisura $\left(t_{1} y t_{2}\right)$. Éstos son:

$$
\mathrm{t}_{1}=\frac{1}{\omega} \arcsin \left(\frac{\sigma_{\mathrm{th}, \mathrm{e}}-\sigma_{m}}{\sigma_{a}}\right)(7 \mathrm{a}) \mathrm{t}_{2}=\frac{\pi-\omega \mathrm{t}_{1}}{\omega}
$$

Por tanto, integrando la ecuación (1) teniendo en cuenta las ecuaciones (2) y (5), se llega a la siguiente expresión:

$$
f=N \int_{t_{1}}^{t_{2}}\left(\sigma_{m}+\sigma_{a} \sin (\omega t)\right)^{n} d t=N h
$$

Donde f es la función definida en la ecuación (4), N el número total de ciclos y $\mathrm{h}$ una función que depende de $\sigma_{\mathrm{m}^{\prime}} \sigma_{\mathrm{a}^{\prime}}, \omega, \mathrm{t}_{1}$, $t_{2}$, y n. Usando la ecuación (3) y teniendo en cuenta que $N=t_{c}$ $\mathrm{n}$, se obtiene el cociente de tiempos de fractura para cada tipo de solicitación, $t_{c} / t_{e}$ :

$$
\frac{t_{c}}{t_{e}}=\frac{2 \pi \sigma_{e}^{n}}{h}
$$

Usando R (ecuación (6)) se puede expresar h como:

$$
\mathrm{h}=\sigma_{\max }(1+R)^{\mathrm{n}} \mathrm{g}
$$

donde la función g se define como:

$$
g=\int_{\omega t_{1}}^{\omega t_{2}}\left(1+\frac{1-R}{1+R} \sin (\omega t)\right)^{n} d(\omega t)
$$

A partir de las relaciones anteriores y teniendo en cuenta que $\sigma_{\mathrm{e}}$ es igual a $\sigma_{\max }$ la ecuación (9) se reescribe como:

$$
\frac{t_{c}}{t_{e}}=\frac{2^{n+1} \pi}{(1+R)^{n} g}
$$

La ecuación (11) no es generalizable porque depende de los parámetros $\omega, t_{1} y_{2}$, relacionados con $v$, el cual varia en cada tipo de ensayo y material. El valor de $\mathrm{n}$ en muchos materiales cerámicos oscila entre 10 y 40, por lo que el valor numérico de la integral g toma valores muy grandes. Así, para un n de 30 si la tensión máxima es superior en un $5 \%$ a $\sigma_{\text {th,e }}$ (variación comparable al error asociado en la medida de $\sigma_{\text {th,e }}$ ), el valor de la integral g es independiente de los límites de integración. Por tanto la ecuación (11) se puede escribir como:

$$
g=\int_{0}^{2 \pi}\left(1+\frac{1-R}{1+R} \sin (\omega t)\right)^{n} d(\omega t)
$$

De esta manera, la integral g sólo depende de n y R. Una vez simplificado el cálculo, se obtienen los cocientes $t_{c} / t_{e}$ para distintos valores de $\mathrm{n}$ y de $\mathrm{R}$ (figura 5). En esta representación se aprecia que al aumentar $R$ disminuye el cociente $t_{c} / t_{e^{\prime}}$ debido a que el material permanece más tiempo en condiciones de crecimiento de fisura, tal y como se ha explicado anteriormente (figura 4). 
Estos cocientes de tiempo permiten estimar el tiempo de fractura bajo cargas fluctuantes para el caso que no exista fatiga cíclica $\left(t_{c}\right)$ a partir de los resultados experimentales bajo carga constante $\left(t_{\mathrm{e}}\right)$. Su aplicación se centra en las curvas $\mathrm{S}-\mathrm{t}$, obteniendo una curva de $\sigma_{\mathrm{F}}$ en función del tiempo para aplicación de cargas cíclicas suponiendo que sólo existe degradación por efecto del medio. La obtención de esta curva se comenta en el apartado 3.4. Cabe destacar que los valores de la integral g obtenidos concuerdan bastante bien con los valores calculados por Evans y Fuller (40). Sin embargo, los valores de $t_{c} / t_{e}$ no son comparables porque en dicho trabajo se supone que $\sigma_{\max }$ es igual a $\sigma_{\mathrm{m}}$.

\subsection{Fisuras de indentación}

\subsubsection{TENSIONES RESIDUALES}

Tal y como se ha comentado este tipo de fisuras son útiles para estudiar el comportamiento mecánico en cerámicas. El problema es que introducen un campo de tensiones residuales difícil de tratar. Así, el factor de intensidad de tensiones consiste en dos términos, el aplicado $\left(\mathrm{K}_{\mathrm{ap}}\right)$ y el residual $\left(\mathrm{K}_{\mathrm{res}}\right)$ (3336):

$$
\mathrm{K}=\mathrm{K}_{\mathrm{ap}}+\mathbf{f} \mathbf{K}_{\mathrm{res}}
$$

Donde $\mathrm{f}$ es un factor reductor de $\mathrm{K}_{\mathrm{res}}$ dado que Shetty et al. (38) han demostrado que las tensiones residuales se reducen al superponer un campo de tensiones aplicado. Desarrollando la ecuación anterior se llega a:

$$
K=Y \sigma \sqrt{c}+f A \frac{P}{c^{3 / 2}}
$$

Siendo c la semilongitud de fisura (figura 1), P la carga aplicada por el penetrador y A una constante que depende del material y que oscila entre 0.016 y $0.024(34,39)$.

Para facilitar la integración de la ecuación (1) con el valor de K de la ecuación (15) se introduce un cambio de variable, $\mathrm{C}=$ $\mathrm{c} / \mathrm{c}_{0}$, siendo $\mathrm{c}_{0}$ la longitud inicial de fisura inducida por el penetrador Vickers. De esta manera la ecuación (15) toma la expresión:

$$
\mathrm{K}=\mathrm{f} \mathrm{K} \mathrm{IC} \mathrm{C}^{-1 / 2}+\mathrm{K}_{\mathrm{ap}, 0} \mathrm{C}^{1 / 2}
$$

Donde $\mathrm{K}_{\mathrm{ap}, 0}$ es el $\mathrm{K}_{\mathrm{ap}}$ inicial, que se escribe como sigue:

$$
\mathrm{K}_{\mathrm{ap}, 0}=\mathrm{Y} \sigma_{\mathrm{ap}} \sqrt{\mathrm{c}_{0}}
$$

\subsubsection{APLICACIÓN DE CARGA CONSTANTE}

La integración de la ecuación (1) para el caso de aplicación de una carga constante $\left(\sigma_{\mathrm{e}}\right)$, y teniendo en cuenta la ecuación (16), lleva a la siguiente relación:

$$
t_{e, i}=\frac{c_{0}}{A} h_{i}
$$

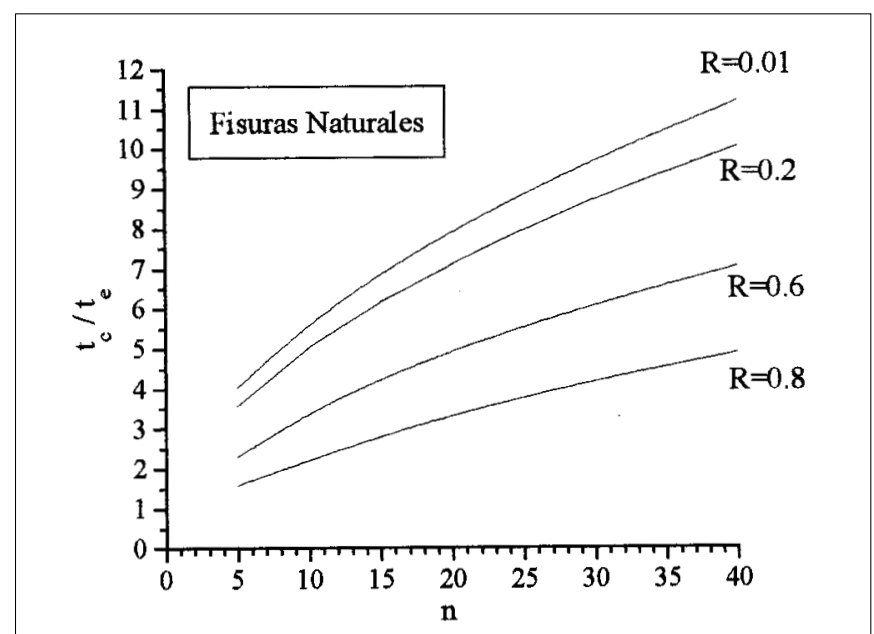

Figura 5: Valores de $t_{c} / t_{e}$ para distintos $n$ y $R$.

Donde $t_{e, i}$ es el tiempo de fractura bajo carga constante de una probeta indentada $y h_{i}$ una función definida como:

$$
h_{i}=\int_{C_{i}}^{C_{f}} \frac{d C}{C^{n / 2}\left(f K_{I C} C^{-2}+K_{a p, 0}\right)^{n}}
$$

\subsubsection{APLICACIÓN DE CARGAS CÍCLICAS}

El caso de cargas cíclicas es más complicado puesto que la combinación de las ecuaciones (4) y (15) convierte la ecuación (1) en una ecuación diferencial de variables no separables de difícil solución. Para abordar este problema se supone, como primera aproximación, que el daño ocasionado por las cargas cíclicas es comparable al producido por una carga estática equivalente $\left(\sigma^{\prime}\right)$. Ésta se calcula de manera que la vida bajo carga constante sea igual que para la aplicación de cargas cíclicas. Así, la resolución de la ecuación (1) teniendo en cuenta la ecuación (16) permite obtener el tiempo de fractura bajo cargas cíclicas $\left(\mathrm{t}_{\mathrm{c}, \mathrm{i}}\right)$ :

$$
t_{\mathrm{c}, \mathrm{i}}=\frac{\mathrm{c}_{0}}{\mathrm{~A}} \mathbf{h}_{\mathrm{i}}{ }^{\prime}
$$

Donde $h_{i}^{\prime}$ se define de forma análoga a $h_{i}$ :

$$
h_{i}^{\prime}=\int_{C_{i}}^{C_{f}} \frac{d C}{C^{n / 2}\left(f K_{I C} C^{-2}+K^{\prime}{ }_{a p, 0}\right)^{n}}
$$

Siendo $\mathrm{K}_{\mathrm{ap}, 0}$ el factor de intensidad de tensiones aplicado inicialmente, donde la carga cíclica ha sido substituida por la carga estática equivalente $\sigma^{\prime}$. La expresión de $\mathrm{K}_{\mathrm{ap}, 0}^{\prime}$ se escribe a continuación:

$$
\mathrm{K}_{\mathrm{ap}, 0}^{\prime}=\mathrm{Y} \sigma^{\prime} \sqrt{\mathrm{c}_{0}}
$$

Por tanto, para calcular los valores de $\mathrm{t}_{\mathrm{e}, \mathrm{i}} \mathrm{y}_{\mathrm{c}, \mathrm{i}}$ a partir de las ecuaciones (18) y (20) es necesario saber los valores del parámetro $\mathrm{f}$ y la longitud de fisura inicial $\mathrm{c}_{0}$. Se ha visto que los valores de f pueden llegar a tener valores de 0.4 para alúmina 
y ZTA (39). Para facilitar el cálculo y evitar tener que calcular $\mathrm{f}$ y $\mathrm{c}_{0}$ para cada caso se introduce los cocientes $\kappa$ y $\kappa^{\prime}$ que los engloban:

$$
\boldsymbol{\kappa}=\frac{\mathbf{f} \mathbf{K}_{\mathrm{c}}}{\mathbf{K}_{\mathbf{a p}, 0}} \quad \text { (23a) } \quad \boldsymbol{K}^{\prime}=\frac{\mathbf{f} \mathbf{K}_{\mathrm{c}}}{\mathbf{K}_{\mathbf{a p}, 0}^{\prime}}
$$

Introduciendo los factores $\kappa$ y $\kappa^{\prime}$ en las ecuaciones (19) y (21), éstas se reescriben como:

$$
\mathrm{h}_{\mathrm{i}}=\frac{\mathrm{h}_{\mathrm{k}}}{\mathrm{K}_{\mathrm{ap}, 0}^{\mathrm{n}}} \quad(24 \mathrm{a}) \quad \mathrm{h}_{\mathrm{i}}{ }^{\prime}=\frac{\mathrm{h}_{\mathrm{k}}{ }^{\prime}}{\left(\mathrm{K}_{\mathrm{ap}, 0}^{\prime}\right)^{\mathrm{n}}}
$$

Donde las funciones $h_{\kappa} y h^{\prime}{ }_{\kappa}$ se definen como:

$$
\begin{aligned}
& h_{\kappa}=\int_{C_{i}}^{C_{f}} \frac{d C}{C^{n / 2}\left(\kappa C^{-2}+1\right)^{n}} \\
& h^{\prime}{ }_{\kappa}=\int_{C_{i}}^{C_{f}} \frac{d C}{C^{n / 2}\left(\kappa^{\prime} C^{-2}+1\right)^{n}}
\end{aligned}
$$

Usando las relaciones anteriores, el cociente de tiempos de fractura $t_{c, i} / t_{e, i}$ se puede expresar como:

$$
\frac{t_{c, i}}{t_{e, i}}=\frac{h_{i}{ }^{\prime}}{h_{i}}=\left(\frac{K_{a p, 0}}{K^{\prime}{ }_{a p, 0}}\right)^{n} \frac{h^{\prime}{ }_{k}}{h_{\kappa}}=\frac{1}{b^{n}} \frac{h^{\prime}{ }_{k}}{h_{\kappa}}
$$

El factor b relaciona $\sigma_{\mathrm{e}}$ y $\sigma^{\prime}$ a través de la ecuación (27) y se ha representado para distintos valores de $\mathrm{n}$ y de $\mathrm{R}$ en la figura 6.

$$
\mathrm{b}=\frac{\mathrm{K}_{\mathrm{ap}, 0}^{\prime}}{\mathrm{K}_{\mathrm{ep}, 0}}=\frac{\sigma^{\prime}}{\sigma_{\mathrm{e}}}
$$

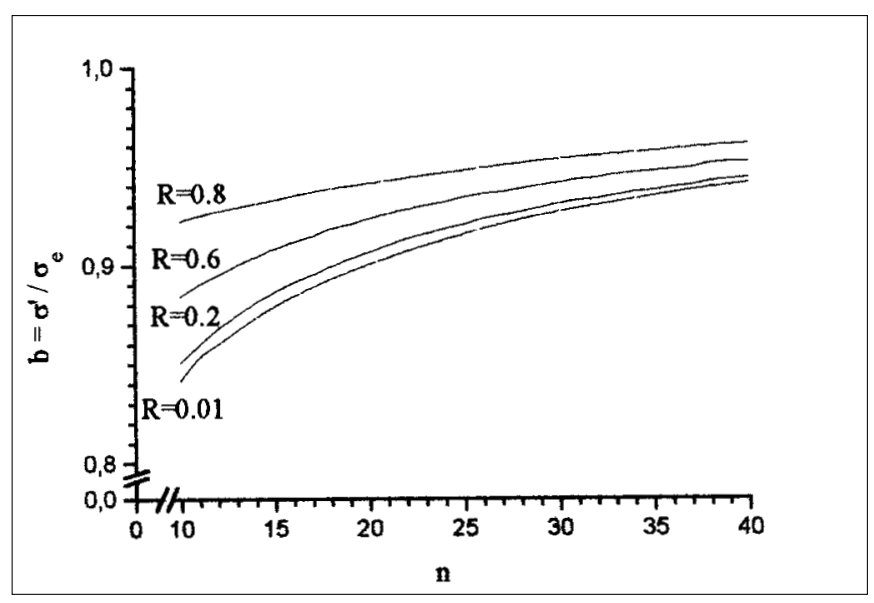

Figura 6: Evolución de b en función de n y de R.

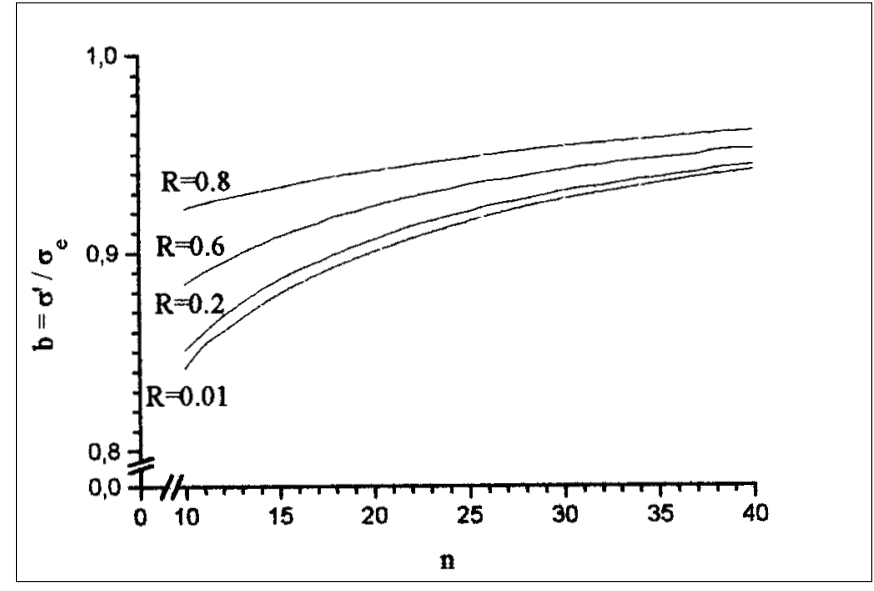

Figura 7: Evolución de $t_{c, i} / t_{e, i}$ en función de $\kappa^{\prime}$ y n, con un valor de $R$ de 0.2 .

Una vez realizados todos los cálculos necesarios se representan gráficamente los valores de $t_{c, i} / t_{e, i}$ en función de $\kappa^{\prime}$ para un $\mathrm{R}$ de 0.2 (figura 7). En esta representación se observa que para valores de $\kappa^{\prime}$ superiores a 0.6 el cociente de tiempos permanece prácticamente constante. En los materiales estudiados (alúmina, mullita y ZTA) se ha visto que con los valores de $\mathrm{f}$ más bajos reportados (f igual 0.4 (39)) el cociente $\kappa$ se acerca a 0.7. Esto permite obtener los valores de $t_{c, i} / t_{e, i}$ a partir de $n y$ de $\mathrm{R}$, sin tener en cuenta ni f ni $\mathrm{K}_{\mathrm{ap}, 0}$ (figura 8). Comparando las figuras 5 y 8 se aprecia que los cocientes de tiempo son menores para el caso de fisuras de indentación. Éstos aumentan progresivamente a medida que las tensiones residuales disminuyen $(\kappa 0)$, hasta llegar a igualarse $a t_{c} / t_{e}$ cuando no hay tensiones residuales $(\kappa=0)$. Una explicación para esta disminución se puede buscar en el incremento del valor del $\mathrm{R}$ efectivo debido a las tensiones residuales (46). Como se aprecia en las figuras 5 y 8 , un aumento en $R$ se traduce en valores de $t_{c} / t_{e} y$ $\mathrm{t}_{\mathrm{c}, \mathrm{i}} / \mathrm{t}_{\mathrm{e}, \mathrm{i}}$ más bajos.

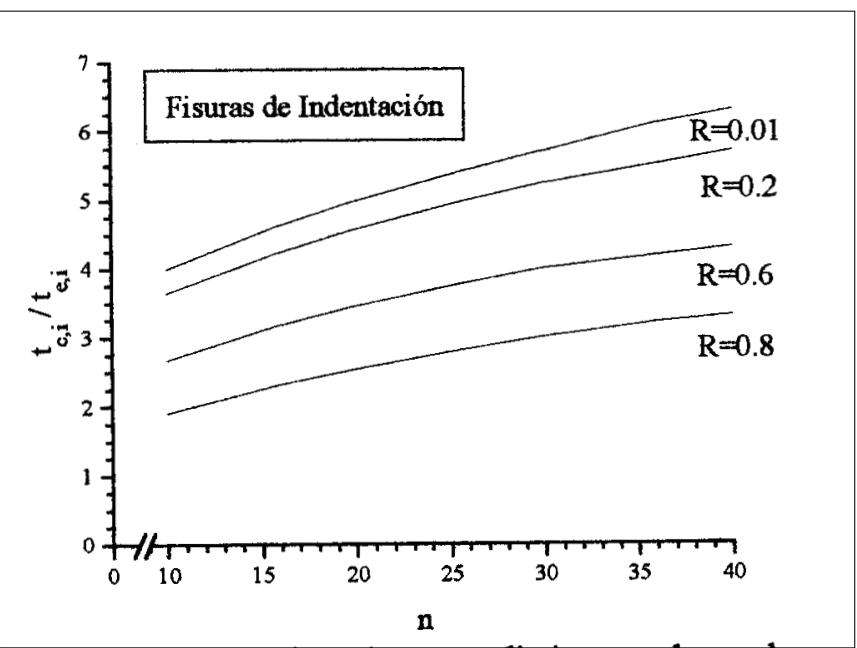

Figura 8: Valores de $t_{c, i} / t_{e, i}$ para distintos valores de $n$ y de $R$ para fisuras de indentación. 


\subsection{Determinación de la presencia de fatiga cíclica}

A partir de los resultados experimentales de fatiga estática se puede estimar la vida a fatiga cíclica si sólo existe degradación por efecto del medio y la carga aplicada, a partir de los cocientes de tiempo calculados en las figuras 5 y 8 . Para obtener estos cocientes es necesario conocer el valor del exponente $\mathrm{n}$ de la ecuación (1). Éste se puede obtener en ensayos de propagación de fisuras grandes, a partir de la pendiente de representaciones doblelogarítmicas da/dt vs K de la ecuación (1). Otro método para obtener $\mathrm{n}$ es a partir de las curvas S-t. Integrando la ecuación (1) para el caso de fisuras de indentación (ecuación (15)) se obtiene una expresión análoga a la ecuación (3). Reescribiendo ésta de otra manera se obtiene una relación entre el esfuerzo $(\sigma)$ y el tiempo de fractura $(t)$ :

$$
\sigma \propto t^{-1 / n^{\prime}}
$$

Donde $\mathrm{n}^{\prime}$ está relacionado con $\mathrm{n}$ en función de la presencia o no de tensiones residuales (1):

$\mathrm{n}^{\prime}=\mathrm{n} \quad$ (no hay tensiones residuales)

$$
\mathrm{n}^{\prime}=\frac{3}{4} \mathrm{n}+\frac{1}{2} \quad \text { (sí hay tensiones residuales) }
$$

Así, los valores de $\mathrm{n}^{\prime}$ se pueden calcular a partir de la pendiente de representaciones doblelogarítmicas tensión-tiempo (curvas S-t), y obtener $\mathrm{n}$ usando las relaciones (28a) o (28b). Una vez calculado el valor de $n$, se obtienen los cocientes $t_{c} / t_{e}$ $o t_{c, i} / t_{e, i}$ en las figuras 5 y 8, según el tipo de fisura, y a partir de ellos se calcula $t_{c}$ o $t_{c, i}$ usando los valores experimentales de $t_{e} o t_{e, i}$. Una vez calculados los valores de $t_{c^{\prime}}$ se pueden obtener las correspondientes curvas S-t para el caso que no exista fatiga mecánica. Si estas curvas se sitúan por encima de los puntos experimentales de fatiga cíclica se puede afirmar que el material sí presenta fatiga cíclica. Por el contrario, si los pun-

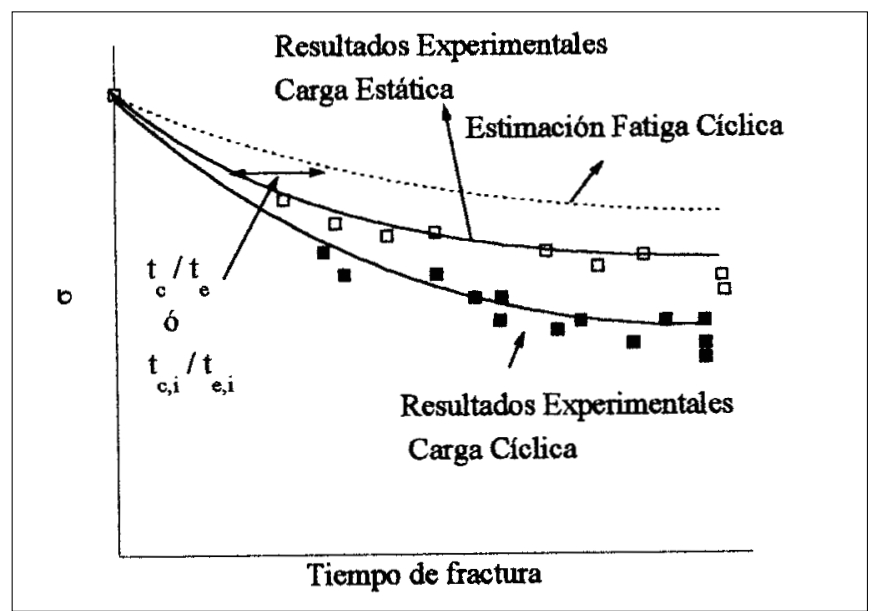

Figura 9: Representación de la obtención de las estimaciones de vida a fatiga a partir de los resultados bajo cargas estáticas. tos experimentales coinciden con la curva estimada, se puede decir que no existe fatiga cíclica en el material. En la figura 9 se esquematizan estas discusiones, mostrándose el caso en que sí existe fatiga cíclica.

Además, a partir de esta estimación también se puede establecer el grado de fatiga cíclica que presenta el material; cuanto más alejados estén los puntos experimentales de la curva
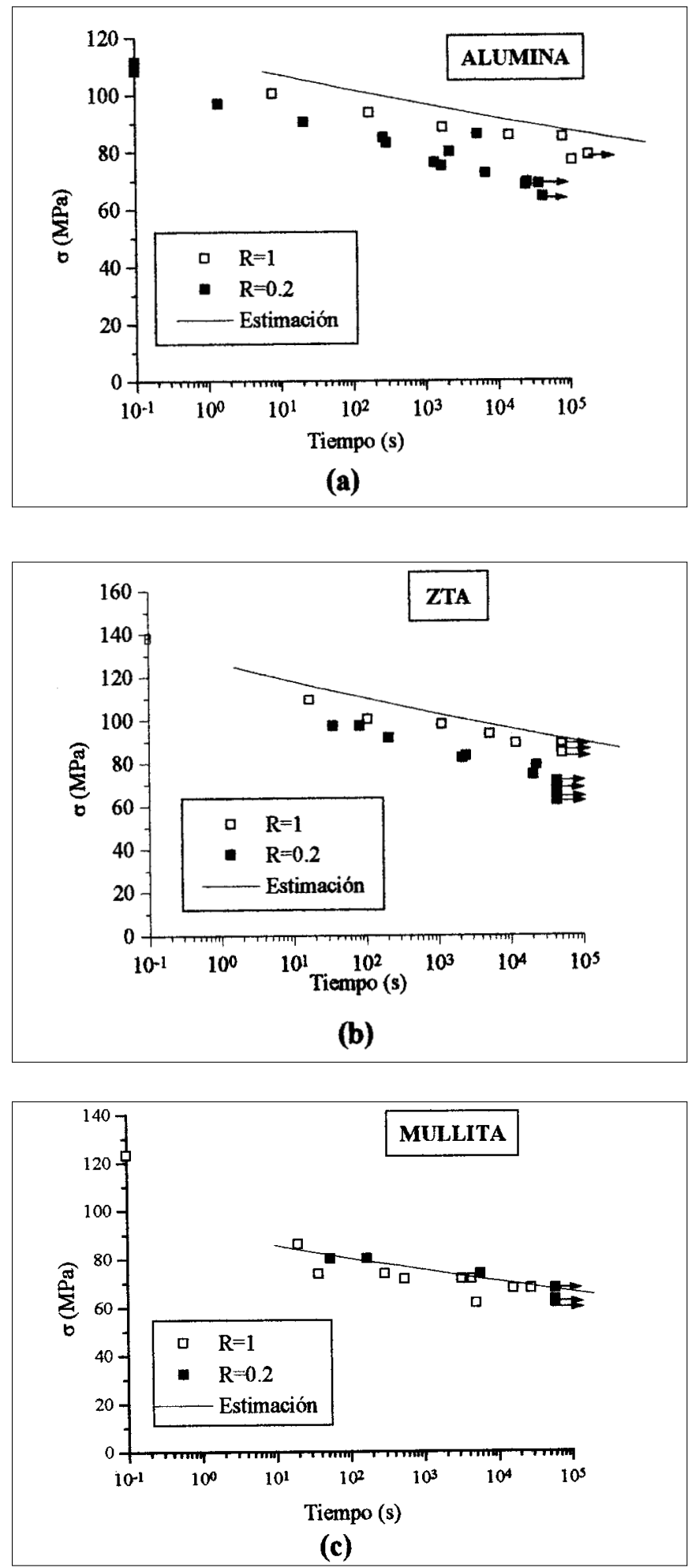

Figura 10: Curvas S-t para los materiales estudiados: (a) alúmina, (b) ZTA y (c) mullita. 
estimada mayor será la degradación introducida en el material por las cargas fluctuantes. Esta susceptibilidad del material a presentar fatiga cíclica se puede relacionar con aspectos microestructurales, a través de los mecanismos de aumento de tenacidad operativos así como a partir del tipo de propagación de fisura (44).

\subsection{Aplicaciones}

La metodología expuesta en el apartado anterior se ha aplicado a los materiales cerámicos citados en la sección 2.1. En todos los casos se han introducido fisuras de indentación con distintas cargas y se ha estudiado el comportamiento bajo cargas constantes ( $\mathrm{R}$ igual a 1 ) y bajo cargas cíclicas, con un $\mathrm{R}$ de 0.2 . Para cada uno de ellos se han realizado las estimaciones de vida a fatiga a partir de los cocientes de tiempo $t_{c, i} / t_{e, i^{\prime}}$ representadas en las curvas S-t como una línea continua (figura 10). Los valores de $\mathrm{n}$ obtenidos a partir de las curvas $\mathrm{S}$-t, siguiendo el proceso descrito en la sección 3.4, son: 40 para alúmina, 33 para ZTA y 30 para mullita.

A partir de las curvas S-t mostradas en la figura 10, se puede establecer si el fenómeno de fatiga mecánica actúa en cada material. Se observa claramente que en alúmina y ZTA los puntos experimentales de fatiga cíclica están por debajo de las estimaciones realizadas; esto denota un marcado efecto de fatiga mecánica en estos materiales. La razón de este comportamiento se encuentra en referencias previas $(15,39)$ y se centra en la degradación del mecanismo de unión de las caras de la fisura actuante en este tipo de materiales Por el contrario, en mullita, los puntos experimentales y las predicciones son muy próximas, señalando que el fenómeno de fatiga mecánica no se detecta en este material. La discusión de este comportamiento se ha realizado en trabajos anteriores (43) y se basa tanto en el carácter transgranular en la propagación de fisuras como en la ausencia de mecanismos de aumento de tenacidad de fractura que puedan ser degradados.

\section{CONCLUSIÓN}

En este trabajo se ha propuesto un método de detección del fenómeno de fatiga cíclica a partir de resultados experimentales de fatiga estática en grietas naturales y de indentación. Los parámetros calculados $\left(t_{c} / t_{e} y_{c, i} / t_{e, j}\right)$ permiten visualizar de manera rápida y directa el grado de fatiga cíclica que presenta el material. En los cálculos realizados se pone de manifiesto que las tensiones residuales acortan las diferencias entre las vidas bajo cargas cíclicas y estáticas cuando sólo existe crecimiento subcrítico por efecto del medio. Esta disminución en los valores de los cocientes de tiempo se explica por el aumento del valor del $\mathrm{R}$ efectivo debido a las tensiones residuales.

\section{AGRADECIMIENTOS}

Este trabajo se ha financiado por el proyecto CICYT MAT 94431. Igualmente se agradece la subvención a esta investigación del ministerio de Educación y Ciencia Español a través del Programa de Cooperación Científica con Iberoamérica (UPCUSB, 1996). Uno de los autores (D.C.) agradece a la Generalitat de Catalunya la beca FI concedida.

\section{REFERENCIAS}

1. B. Lawn, Fracture Mechanics of Brittle Solids, Cambridge University Press, U.K. (1995).

2. A.G. Evans, "Perspective on the Development of High-Toughness Ceramics", J. Am. Ceram. Soc. 73 (2) 187-206 (1992).

3. D.J. Green, R.H.J. Hannink y M.V. Swain, Transformation Toughening of Ceramics, CRC Press Inc., Florida (1989).

4. P. Chantikul, S.J. Bennison y B.R. Lawn, "Role of Grain Size in the Strength and R-Curve Properties of Alumina", J. Am. Ceram. Soc. 73 (8) 2419-27 (1990).

5. E. Orowan, "The Fatigue of Glass Under Stress", Nature 154 341-3 (1944).

6. S.M. Widerhorn y L.H. Bolz, "Stress Corrosion as Static Fatigue of Glass", J. Am. Ceram. Soc. 53 (10) 543-8 (1970) .

7. S. W. Freiman, D.R. Mulville y P.W. Mast, "Crack Propagation Studies in Brittle Materials", J. Mater Sci. 8 1527-33 (19).

8. S.M. Widerhorn, H. Johnson, A.M. Diness y A.H. Heuer, “Fracture of Glass in Vacuum", J. Am. Ceram. Soc. 57 (8) 336-41 (1974).

9. P.F. Becher, "Slow Crack Growth Behaviour in Transformation-Toughened $\mathrm{Al}_{2} \mathrm{O}_{3}-\mathrm{ZrO}_{2}\left(\mathrm{Y}_{2} \mathrm{O}_{3}\right)$ Ceramics", J. Am. Ceram. Soc. 66 (7) 485-8 (1983).

10. P.F. Becher, "Subcritical Crack Growth in Partially Stabilized $\mathrm{ZrO}_{2}(\mathrm{Mg}$ PSZ)", J. Mater. Sci. 21 297-300 (1986).

11. T. Sato, T. Endo, M. Shimada, T. Mitsudone y N. Otabe, "Hydrothermal Corrosion of Magnesia-Partially-Stabilised Zirconia", J. Matter. Sci. 26 1346-1350 (1991).

12. J. Drennan, A. J. Harstshorn y S.W. Thompson, "The Effect of Low temperature Aqueous Environments on Magnesia-Partially-Stabilised Zirconia (MgPSZ)", pp.145-51 en Advances in Ceramics: Science and Technology of Zirconia V, (Eds. S.P.S. Badwal, M.J. Bannister y R.H.J. Hannink), Am. Ceram. Soc., Columbus OH. 1993.

13. J. Alcalá, "Propagación Subcrítica de Grietas bajo Cargas Cíclicas, Constantes y Monotónicas en la Y-TZP", Tesis Doctoral, Universitat Politècnica de Catalunya (1994)

14. H. Yin, M. Gao y R.P. Wei, "Phase Transformation and Sustained Load Crack Growth in $\mathrm{ZrO}_{2}+3 \mathrm{~mol} \% \mathrm{Y}_{2} \mathrm{O}_{3}$ : Experiments and kinetic modelling", Acta metall. mater. 43 (1) 371-82 (1995).

15. M.M. Nagl, D. Casellas, L. Llanes, C. Domínguez y M. Anglada., “Fatiga Estática y Cíclica de Alúmina reforzada con Partículas de Circona", Anales de Mecánica de la Fractura Vol. 12, 349-54 (1995).

16. T.A. Michalske y S.W Freiman, "A Molecular Mechanism for Stress Corrosion in Vitreous Silica", J. Am. Ceram. Soc. 66 (4) 284-8 (1983).

17. R. Thompson, "The Molecular Wedge in a Brittle Crack: A simulation of mica/ water", J. Mater. Res. 5 (3) 524-34 (1990).

18. A.G. Evans, "Fatigue in Ceramics", Int J. Frac.16 485-98 (1980).

19. D.A Krohn y D.P.H. Hasselman, "Static and Cyclic Fatigue Behaviour of a Polycrystalline Alumina", J. Am. Ceram. Soc. 55 208-11 (1972).

20. F. Guiu, "Cyclic fatigue of Polycrystalline Alumina in Direct Push-Pull”, J. Mater. Sci. Lett. 13 1357-61 (1978).

21. R.O. Ritchie y K.T Venkateswara Rao, "Cyclic Fatigue-Crack Growth in Toughened Ceramics and Intermetallics at Ambient to Elevated Temperatures", pp.53-69 en ECF 11-Mechanisms and Mechanics of Damage and Failure, Ed. J. Petit (1996).

22. S. Lathabai, J. Rödel y B. Lawn, "Cyclic Fatigue from Frictional Degradation of Bridging Grains in Alumina", J. Am. Ceram. Soc. 74 (6) 1340-8 (1991).

23. F. Guiu, M. Li y M.J. Reece, "Role of Crack-Bridging Ligaments in the Cyclic Fatigue Behaviour of Alumina", J. Am. Ceram. Soc. 75 (11) 2976-84 (1992).

24. M.J. Hoffman, R.H. Dauskardt, Y.-W Mai y R.O. Ritchie, "A Review of the Mechanics and Mechanisms of Cyclic Fatigue-Crack Propagation in Transformation-Toughened Zirconia Ceramics", pp. 321-38 en Advances in Ceramics: Science and Technology of Zirconia V, (Eds. S.P.S. Badwal, M.J. Bannister y R.H.J. Hannink), Am. Ceram. Soc., Columbus OH. 1993.

25. R.H. Dauskardt, D.B. Marshall y R.O. Ritchie, "Cyclic Fatigue Crack Propagation in Magnesia-Partially-Stabilised Zirconia Cera-mics", J. Am. Ceram. Soc. 73 (4) 893-903 (1990).

26. M. Hoffman, Y.-W. Mai, S. Wakayama, M. Kawahara y T. Kishi. “Crack-Tip Degradat-ion Processes Observed During in situ Cyclic Fatigue of Partially Stabilised Zirconia", J. Am. Ceram. Soc. 78 (10) 2801-10 (1995).

27. F. Guiu, M.J. Reece y D.A.J. Vaughan, "Cyclic Fatigue in Some Structural Ceramics", pp.193-210 en Proc. of the Eng. Foundation Conf. on Fatigue of Advanced Materials. UK: Mat.\&Component Eng. Publ. Ltd.Eds. Ritchie R.O., Dauskardt R.H y B.N. Cox. (1991). 
28. S. Suresh y J.R. Brockenbrough, "Theory and Experimentals of fracture in cyclic compression: single phase ceramics, transforming ceramics and ceramic composites", Acta metall. 36 1455- (1988).

29. M.M. Nagl, L. Llanes, R. Fernández y M. Anglada, “The Fatigue Behaviour of Mg-PSZ and ZTA Ceramics", pp. 61-76 en Fracture Mechanics of Ceramics Vol 12: Fatigue, Composites and High Temperature Behaviour. Karlsruhe (Alemania), 1994. Eds. R.C. Bradt, D.P.H. Hasselman, D. Munz. M. Sakai y V. Ya, Plenum. 1996.

30. W.E.C. Creyke, I.E.J. Sainsbury y R. Morell, Design with Non-Ductile Materials, Applied Science Publishers LTD. (1982).

31. B.R. Lawn y T.R. Wilshaw, "Indentation Fracture: Principle and Applications", J. Mater. Sci. 10 (6) 1049-81 (1975).

32. B.R. Lawn, A.G. Evans y D.B. Marshall, "Elastic/plastic Indentation Damage in Ceramics: The Medium/Radial Crack System", J. Am. Ceram. Soc. 63 (9-10) 574-81 (1980).

33. D.B. Marshall y B.R. Lawn, "Residual Stress Effects in Sharp-Contact Cracking", J. Matter. Sci. 14 (8) 2001-12 (1979)

34. G.R. Anstis, P. Chantikul, B.R. Lawn y D.B. Marshall, "A Critical Evaluation of Indentation Techniques for Measuring Fracture Toughness: I, Direct Crack Measurements", J. Am. Ceram. Soc. 64 (9) 533-8 (1981)

35. G.R. Anstis, P. Chantikul, B.R. Lawn y D.B. Marshall, "A Critical Evaluation of Indentation Techniques for Measuring Fracture Toughness: II, Strength Methods", J. Am. Ceram. Soc. 64 (9) 538-46 (1981).

36. M. Sakai y R.C. Bradt. "Fracture Toughness Testing of Brittle Materials", International Materials Review 38 53-78 (1993).

37. J.C. Newman y I.S. Raju. “An Empirical Stress-Intensity Factor Equation for the Surface Crack”. Eng. Frac. Mec. 15 (1-2) 185-92 (1981)
38. D.K. Shetty , A.V. Virkar, A.R. Rosenfield y W. Duckworth, "A 'Compressed-Spring' Analogy for Residual Stress Effects on the Extension of Indenter Flaws", J. Am. Ceram. Soc. 67 (8) C201-C203 (1991).

39. D. Casellas, M.M. Nagl, L. Llanes y M. Anglada, "Growth of Small Surface Indentation Cracks in Alumina and Zirconia Toughened Alumina", Key Engin. Mater., Vol 127-131, Part 2 , 895-902 (1996).

40. A.G. Evans y E.R. Fuller, “Crack Propagation in Ceramic Materials Under Cyclic Loading Conditions", Met. Trans. 5 27-33 (1974).

41. T. Fett y D. Munz, "Subcritical Crack Extension in Ceramics", Frac. Mec. 5 505-23 (1989).

42. M.J. Hoffman, W. Lentz, M.V. Swain y Y-W. Mai, “Cyclic Fatigue Lifetime Prediction of Partially Stabilised Zirconia with Crack Resistance Curve Characteristics", J. Eur. Ceram. Soc. 11 445-53 (1993).

43. D. Casellas, C. Baudín, M. Osendi, L. Llanes y M. Anglada, “Influencia de la Microestructura en la Fatiga de Mullita", Anales de Mecánica de la Fractura Vol. 14, 200-5 (1997).

44. D. Casellas, R. Fernández, M.M. Nagl, J. Alcalá, L. Llanes y M. Anglada, "Influencia de la Distribución y Morfología de las Fases en la Susceptibilidad a Fatiga de Materiales Cerámicos", Anales de Mecánica de la Fractura Vol. 14, 20611 (1997).

45. M. I. Osendi y C. Baudín "Mechanical Properties of Mullite Materials", J. Eur. Ceram. Soc., 16 217-24 (1996).

46. D. Casellas, L. Llanes, M.M. Nagl y M. Anglada, "Influence of Indentation Residual Stresses on the Cyclic Fatigue Limit of Ceramic Materials", en Cinquième Colloque Interregional Europeen sur les Ceramiques CIEC 5, Toulon (Francia), (1996), en publicación.

Recibido: 10-6-97

Aceptado: 2-12-98

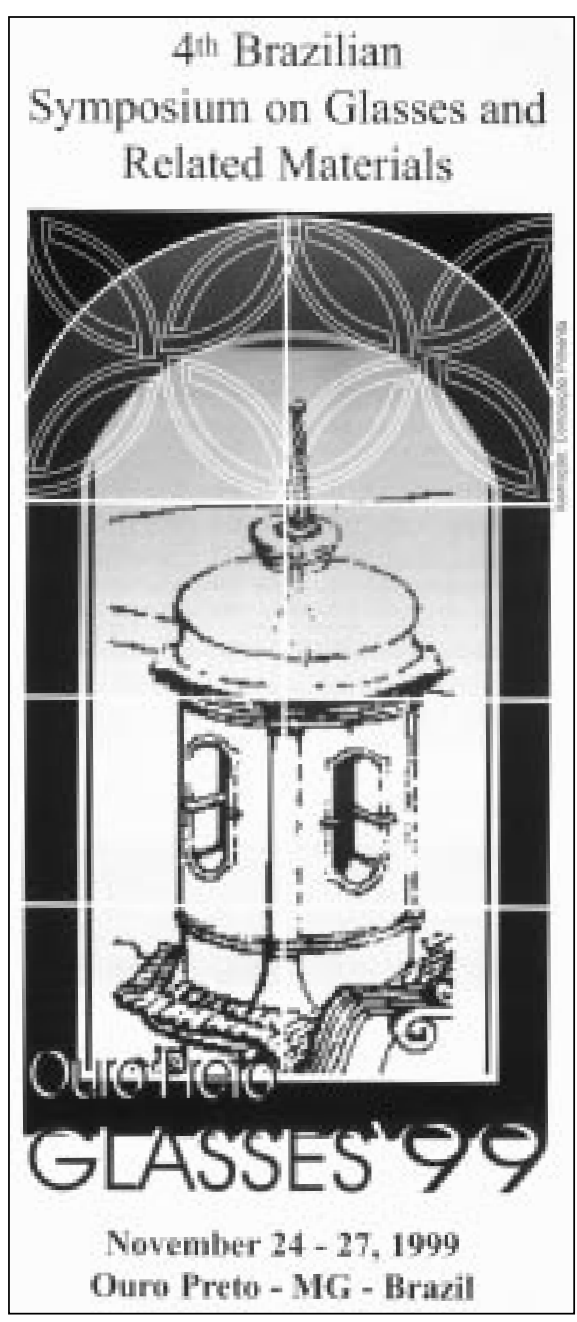

\section{INFORMATION AND CORRESPONDENCE}

Prof. Dr. Wander L. Vasconselos, Chairman Universidade Federal de Minas Gerais Depto. de Engenharia Metalúrgica e de Materiais Laboratório de Materiais Cerâmicos - LMC

Rua Espírito Santo 35, 2o andar CEP 30160-030 Belo Horizonte - MG, Brazil Fone: 55-31-238-1821 - Fax: 55-31-238-1815 E-mail: glass99@urano.cdtn.br

\section{Dra. Vilam C. Costa Secretary}

Centro de Desenvolvimiento da Tecnologia Nuclear

Caixa Postal 941 - Cidade Universitária CEP 30.123-970 Belo Horizonte - MG, Brazil

Fone: 55-31-499-3447 - Fax: 55-31-499-3390 E-mail: glass99@urano.cdtn.br 